\title{
DNA methylation profiling in recurrent miscarriage
}

\author{
Li Pi ${ }^{\text {Equal first author, } 1}$, Zhaofeng Zhang ${ }^{\text {Equal first author, } 1}{ }^{\text {, Yan }} \mathbf{G u}^{2}$, Xinyue Wang ${ }^{1}$, Jianmei Wang ${ }^{2}$, Jianhua Xu ${ }^{1}$, Junwei Liu ${ }^{1}$, \\ Xuan Zhang ${ }^{\text {Corresp., } 1}$, Jing Du ${ }^{\text {Corresp. } 1}$ \\ ${ }^{1}$ NHC Key Lab. of Reproduction Regulation $\square$ Shanghai Institute of Planned Parenthood Research), Medical School, Fudan University, Shanghai, China \\ 2 The Second Hospital of Tianjin Medical University, Tianjin, China \\ Corresponding Authors: Xuan Zhang, Jing Du \\ Email address: xuanzhang@sippr.org.cn, dujing42@126.com
}

Recurrent miscarriage (RM) is a complex clinical problem. However, specific diagnostic biomarkers and candidate regulatory targets have not yet been identified . To explore RMrelated biological markers and processes, we performed a genome-wide DNA methylation analysis using the Illumina Infinium HumanMethylation450 array platform. Methylation variable positions and differentially methylated regions (DMRs) were selected using the Limma package in R language. Thereafter, gene ontology (GO) enrichment analysis and pathway enrichment analysis were performed on these DMRs. A total of 1799 DMRs were filtered out between patients with RM and healthy pregnant women. The GO terms were mainly related to system development, plasma membrane part, and sequence-specific DNA binding, while the enriched pathways included cell adhesion molecules, type I diabetes mellitus, and ECM-receptor interactions. In addition, genes, including $A B R$, ALCAM, HLA-E, HLA-G, and ISG15, were obtained. These genes may be potential candidates for diagnostic biomarkers and possible regulatory targets in RM. We then detected the mRNA expression levels of the candidate genes. The mRNA expression levels of the candidate genes in the RM group were significantly higher than those in the control group. However, additional research is still required to confirm their potential roles in the occurrence of RM. 


\section{DNA methylation profiling in recurrent miscarriage}

2 Li Pi ${ }^{1 \#}$, Zhaofeng Zhang ${ }^{1 \#}$, Yan $\mathrm{Gu}^{2}$, Xinyue Wang ${ }^{1}$, Jianmei Wang ${ }^{2}$, Jianhua Xu ${ }^{1}$, Junwei Liu ${ }^{1}$,

3 Xuan Zhang ${ }^{1 *}$, Jing $\mathrm{Du}^{1^{*}}$

4

5 1. NHC Key Lab. of Reproduction Regulation(Shanghai Institute of Planned Parenthood

6 Research), Medical School, Fudan University, Shanghai 200032, China

7 2. The Second Hospital of Tianjin Medical University, Tianjin, China

$8 \quad$ \# contributed equally

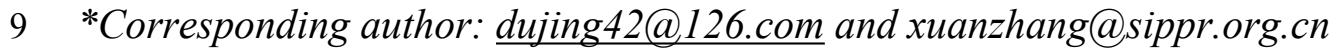




\section{Abstract}

12 Recurrent miscarriage $(\mathrm{RM})$ is a complex clinical problem. However, specific diagnostic

13 biomarkers and candidate regulatory targets have not yet been identified. To explore RM-related

14 biological markers and processes, we performed a genome-wide DNA methylation analysis using

15 the Illumina Infinium HumanMethylation450 array platform. Methylation variable positions and

16 differentially methylated regions (DMRs) were selected using the Limma package in R language.

17 Thereafter, gene ontology (GO) enrichment analysis and pathway enrichment analysis were

18 performed on these DMRs. A total of 1799 DMRs were filtered out between patients with RM and

19 healthy pregnant women. The GO terms were mainly related to system development, plasma membrane part, and sequence-specific DNA binding, while the enriched pathways included cell adhesion molecules, type I diabetes mellitus, and ECM-receptor interactions. In addition, genes, including $A B R, A L C A M, H L A-E, H L A-G$, and $I S G 15$, were obtained. These genes may be potential candidates for diagnostic biomarkers and possible regulatory targets in RM. We then detected the mRNA expression levels of the candidate genes. The mRNA expression levels of the candidate genes in the RM group were significantly higher than those in the control group. However, additional research is still required to confirm their potential roles in the occurrence of RM. 
Introduction

Recurrent miscarriage (RM), defined as two or more consecutive clinically recognized spontaneous pregnancy losses before 20 weeks of gestation (Rai \& Regan 2006), affects $1-5 \%$ of women within the reproductive age (Kim et al. 2004; Pildner \& Ktm 2009). Multiple causes have been found to contribute to the pathogenesis of RM, including chromosomal abnormalities, cervical incompetence, uterine anomalies, autoimmune diseases, endocrinological abnormalities, antiphospholipid antibodies, thrombophilic disorders, low progesterone levels, and microbial infections (Hou et al. 2016; Rai \& Regan 2006). Approximately 40-50\% of cases remain unexplained ( $\mathrm{Li}$ et al. 2002); however, the molecular mechanisms have not been fully identified (Griebel et al. 2005). Determining potential diagnostic biomarkers and possible regulatory targets of RM may help promote research progress. Therefore, new methods are needed.

Recently, there has been an increasing interest in the role of epigenetic mechanisms in human diseases. One promising approach is DNA methylation profiling. DNA methylation, a wellcharacterized epigenetic modification, is critical for development and differentiation ( $\mathrm{Li}$ et al. 1992; Ziller et al. 2013). In addition, it has been proposed that DNA methylation may be an important factor in the regulation of gene expression, $\mathrm{X}$ chromosome inactivation, genomic imprinting, chromatin modification, endogenous retrovirus silencing, and developmental origins of common human diseases (Bestor 2000; Bird \& Wolffe 1999; Reik \& Walter 2001; Takai \& Jones 2002). By using DNA methylation profiling, we can obtain adequate information on aberrant DNA methylation events (Yagi et al. 2008).

In addition, a previous study has established that DNA methylation occurs during early embryonic 
49 development (Li 2002). Aberrant DNA methylation, arising during embryonic development, has

50 been identified as a potential cause of pregnancy loss (Hanna et al. 2013). Accordingly, we mainly

51 focused on the genes involved in embryonic development to identify the biomarkers of RM.

52 In the present study, we used the Illumina Infinium HumanMethylation450 array platform to

53 conduct a genome-wide screening of DNA methylation in decidua samples from the products of

54 conception of women with RM and to identify novel methylation variable positions (MVPs) and

55 differentially methylated regions (DMRs). Furthermore, to gain insight into the molecular

56 regulatory mechanisms of RM, gene ontology (GO) and pathway enrichment analyses were used

57 to explore the potential diagnostic biomarkers and possible regulatory targets. The mRNA

58 expression levels of candidate genes were detected using real-time PCR.

59 The contribution of errors to miscarriage and whether the DNA methylation process plays a pivotal

60 role in fetal programming have not been well explored. In this study, we aimed to evaluate the

61 association of aberrant DNA methylation between patients with RM and healthy pregnant women

62 and obtain data for further understanding human pregnancy loss by predicting potential RM-

63 related biological processes and pathways, as well as candidate genes. 


\section{Materials and methods}

66

67

68

69

\section{Samples}

We enrolled 15 healthy pregnant women and 15 patients with RM from the outpatient department of Gynecology and Obstetrics, The Second Hospital of Tianjin Medical University, China. All participants were recruited in accordance with the same inclusion and exclusion criteria. The participants were considered to have RM if they had at least two consecutive miscarriages. The participants were considered as controls if they had at least one live birth and no history of miscarriage, still birth, preterm labor, or pre-eclampsia and if their pregnancy was terminated for non-medical reasons or they underwent legal abortions. The exclusion criteria included endocrine diseases, infections, chromosomal abnormalities, immunological diseases, and anatomical abnormalities of the genital tract. Gestational weeks ranged from 6 to 12 weeks, and the maternal age ranged from 22 to 42 years (Table 1). Decidual tissue was collected anonymously via curettage in The Second Hospital of Tianjin Medical University from June 2013 to August 2013. All collected tissues were fragmented and stored immediately at $-80^{\circ} \mathrm{C}$ in RNAlater solution (Invitrogen, Carlsbad, CA) until RNA and DNA extraction. Written informed consent was obtained from all patients who provided tissue samples, and consent to publish research data derived from these collected samples was also obtained. The collection of the samples for this study was approved by the Shanghai Institute of Planned Parenthood Research Clinical Ethics Review Board (No.PJ2015). 
86

87

According to the manufacturer's protocol, genomic DNA was extracted from the decidual tissues using the DNeasy Blood and Tissue Kit (TransGen, China) after the samples were digested via proteinase $\mathrm{K}$ and treated with RNase.

\section{Infinium HumanMethylation450 BeadChip processing}

DNA from each sample was treated with sodium bisulfate and processed for analysis on the Illumina Infinium HumanMethylation450 array platform at Genergy (Shanghai, China). Before proceeding to the statistical analysis, all data were processed via Beta Mixture Quantile dilation. Linear models were developed to calculate P-values using the Limma package of the R software (Smyth 2005). After Benjamini and Hochberg correction, we screened significant MVPs. Meanwhile, using the Probe Lasso method of the ChAMP package, we estimated the DMRs. Volcano plot and heat map software programs were used to analyze and visualize the data.

\section{GO enrichment analysis}

GO analysis was utilized to explain the primary DMR function based on the GO database, which is the crucial functional classification database of the NCBI (Ashburner et al. 2000; Gene Ontology 2006). Fisher's exact test was used to calculate the significance level (P-value) of each GO term to screen out the significant GO terms of DMR enrichment. P-values $<0.05$ were considered to be statistically significant.

\section{Pathway enrichment analysis}

Pathway analysis was performed to determine the significant pathway terms of the DMRs according to the Kyoto Encyclopedia of Genes and Genomes (KEGG) (Kanehisa \& Goto 2000).

We used Fisher's exact test to identify significant pathway terms, and P-values $<0.05$ were also 
107 considered statistically significant (Draghici et al. 2007; Kanehisa et al. 2004).

\section{Quantitative real-time PCR}

109 The total RNA of the decidual tissues was extracted using the EasyPure RNA Kit (TransGen

110 Biotech, Beijing, China). Reverse transcription reaction was performed with 500 ng of total RNA

111 using the PrimeScript RT Master Mix Perfect Real Time Kit (TaKaRa, Japan). cDNA (2 $\mu$ L) was

112 added to the $18 \mu \mathrm{L}$ reaction mixture containing $6 \mu \mathrm{L}$ of $\mathrm{ddH}_{2} \mathrm{O}, 10 \mu \mathrm{L}$ of SYBR Premix Ex Taq

113 II, $0.4 \mu \mathrm{L}$ of ROX Reference Dye or Dye II (TaKaRa), and $0.8 \mu \mathrm{L}$ of each primer. The PCR

114 conditions were as follows: $95^{\circ} \mathrm{C}$ for $1 \mathrm{~min}$, followed by 40 cycles at $95^{\circ} \mathrm{C}$ for $15 \mathrm{~s}, 58^{\circ} \mathrm{C}$ for $20 \mathrm{~s}$,

115 and $72^{\circ} \mathrm{C}$ for $20 \mathrm{~s}$, and a final extension at $72^{\circ} \mathrm{C}$ for $5 \mathrm{~min}$. All samples were assayed in triplicate.

116 Relative gene expression levels were calculated using the $2^{-\Delta \Delta \mathrm{Ct}}$ method. 
118

119

120

121

122

123

124

125

126

127

128

129

130

131

132

133

134

135

136

137

138

\section{Results}

\section{Volcano plot and heat map of the MVPs}

We used the R software to screen the MVPs. Using the algorithms provided in the Limma package after Benjamini and Hochberg correction, we collected data and produced a volcano plot and heat map.

The volcano plot, generated using the volcano plot function, showed the results of the MVPs between the controls and patients with RM. The dots in the upper left section denoted the hypomethylated loci, and those in the upper right section denoted the hyper-methylated loci (Figure 1a). The heat map, generated using the heatmap function, showed the hierarchical clustering of the MVPs between the controls and patients with RM (Figure 1b).

The MVP analysis indicated that C2orf54, CECR2, TMEM161B, ABR, EMCN, TBCD, GP5, PRICKLE2-AS3, NBPF22P, and TBX3 showed differential methylation levels with respect to the cases and controls (Table 2). Among these genes, we screened CECR2 (P=1.7299E-06), ABR $(\mathrm{P}=3.55356 \mathrm{E}-06)$, and $\mathrm{TBX} 3(\mathrm{P}=6.52408 \mathrm{E}-06)$, which are closely related to embryonic development, as candidate genes of RM (Banting et al. 2005; Chen et al. 2010; Esmailpour \& Huang 2012; Ohgushi et al. 2017).

\section{GO enrichment analysis and GO-Tree of the DMRs}

To conduct a functional enrichment analysis to identify RM-related biological processes, cellular components, and molecular functions, GO enrichment analysis of 1799 DMRs was conducted. The most enriched biological processes as determined in the GO term analysis were associated with system development (GO:0048731, $\mathrm{P}=1.86 \mathrm{E}-30)$, multicellular organismal development 
139 (GO:0007275, $\mathrm{P}=1.05 \mathrm{E}-29)$, and anatomical structure development (GO:0048856, $\mathrm{P}=4.62 \mathrm{E}-28)$.

140 Within the cellular component category, the most enriched GO terms were significantly associated

141 with the plasma membrane part (GO:0044459, P=9.42E-11), plasma membrane (GO:0005886,

$142 \mathrm{P}=1.86 \mathrm{E}-09)$, and cell periphery $(\mathrm{GO}: 0071944, \mathrm{P}=4.57 \mathrm{E}-09)$. In the molecular function category,

143 the GO terms enriched for the DMRs in RM included sequence-specific DNA binding

144 (GO:0043565, $\mathrm{P}=1.61 \mathrm{E}-24)$, sequence-specific DNA binding transcription factor activity

145 (GO:0003700, $\mathrm{P}=7.89 \mathrm{E}-21$ ), and nucleic acid binding transcription factor activity (GO:0001071,

$146 \mathrm{P}=9.29 \mathrm{E}-21)$ (Figure 2a, Table 3).

147 The GO enrichment analysis for the DMRs indicated that the genes were mainly enriched in the

148 GO terms, including HES4, PRKCZ, SKI, PRDM16, ALPL, RUNX3, TAL1, TTLL7, BARHL2,

149 OLFM3, NFYC, TBX15, LMX1A, TNFRSF4, AJAP1, SYTL1, OPRD1, PTPRU, PRRX1, ELF3,

$150 P R O X 1, H L A-G$, and $H L A-E$ (Table 4). Among these, we screened $H L A-G(\mathrm{P}=1.86 \mathrm{E}-30), H L A-E$

$151(\mathrm{P}=9.42 \mathrm{E}-11)$, and PRDM16 $(\mathrm{P}=1.86 \mathrm{E}-30)$, which are closely related to embryonic development,

152 as candidate genes of RM (Gelmini et al. 2016; Horn et al. 2011; Verloes et al. 2017).

153 To determine the intrinsic link among gene functions, hierarchical trees were constructed. The

154 results showed that the genes were closely related to development, plasma, and DNA binding in

155 RM (Figure 2b,2c,2d).

\section{Pathway enrichment analysis of the DMRs}

157 To generate further insight into the pathways, we performed KEGG pathway annotation to

158 determine whether significant DMRs were enriched for any pathway. From the results, the top five

159 frequently enriched pathways were as follows: cell adhesion molecules (CAMs) 
160 (PATHWAY:hsa04514, P=3.00E-08), type I diabetes mellitus (PATHWAY:hsa04940, P=1.27E-

161 06), ECM-receptor interaction (PATHWAY:hsa04512, P=4.24E-06), allograft rejection

162 (PATHWAY:hsa05330, 1.02E-05), and antigen processing and presentation

163 (PATHWAY:hsa04612, p=2.05E-05) (Figure 3, Table 5).

164 The KEGG pathway enrichment analysis for the DMRs indicated the mainly enriched genes,

165 which included CADM3, F11R, NRXN1, ITGA4, PDCD1, ALCAM, NLGN1, CLDN16, HLA-G,

166 HLA-E, HLA-B, TNF, HLA-DQB1, COL11A1, SV2A, LAMB3, ITGA4, and COL6A3 (Table 6).

167 Among these, we screened $A L C A M(\mathrm{P}=3.00 \mathrm{E}-08), H L A-G(\mathrm{P}=3.00 \mathrm{E}-08)$, and $H L A-E(\mathrm{P}=3.00 \mathrm{E}-$

168 08), which are closely related to embryonic development, as candidate genes of RM (Cizelsky \&

169 Tata 2014; Gelmini et al. 2016; Verloes et al. 2017).

$m R N A$ relative expression level of the candidate genes in 24 samples

171 Based on the abovementioned processing and analysis data, we selected five genes (ISG15, $A B R$,

$172 H L A-E, H L A-G$, and $A L C A M$ ) with significant differences in methylation expression and possible

173 relationships to embryogenesis and development for quantitative real-time PCR verification. The

174 analysis showed that the mRNA expression levels of $I S G 15, A B R, H L A-E$, and $H L A-G$ were higher

175 in the RM group than in the control group $(\mathrm{P}<0.05)$. There was no significant difference in the

176 mRNA expression level of $A L C A M$ between the two groups $(\mathrm{P}>0.05)$ (Figure 4). 


\section{Discussion}

Previous studies have attempted to determine the association between the DNA methylation status and mechanisms that lead to RM. Some studies have suggested that MTHFR is a candidate gene that plays an important role during pregnancy by regulating thrombotic events or methylation (Mishra et al. 2019). Other studies have shown that increasing FOXP3 promoter methylation levels may cause abnormal immune tolerance through downregulation of the expression of FOXP3 protein, which consequently leads to unexplained recurrent spontaneous abortion (Hou et al. 2016). A study in which combined analysis of DNA methylation and gene expression was performed showed CREB5 as a contributor in RM (Yu et al. 2018). Moreover, abnormal methylation of the decidua has been demonstrated to be associated with pregnancy failure in an animal model (Brown et al. 2013). In this study, we analyzed the DNA methylation profile of MVPs and DMRs in decidua samples obtained from 15 patients with RM and 15 controls via a genome-wide DNA methylation analysis using the microarray platform, Illumina Infinium HumanMethylation450 BeadChip. Furthermore, we were able to conduct GO enrichment analysis and pathway enrichment analysis of the DMRs. In the GO enrichment analysis, system development, plasma membrane part, and sequence-specific DNA binding were the most related GO terms. In the pathway enrichment analysis, the DMRs were mainly involved with CAMs, type I diabetes mellitus, and ECM-receptor interaction. In addition, we proposed that aberrant methylation of novel genes, $A B R$, $A L C A M, H L A-E, H L A-G$, and $I S G 15$, may be crucial for embryonic progress and development and that these may serve as candidate genes closely related to RM.

Among the top ranked genes in the MVPs, $A B R$ is likely to be the candidate loci of RM. ABR, a 
199

200

201

202

203

204

205

206

207

208

209

210

211

212

213

214

215

216

217

218

219

regulator of Rho-family small GTPases, has been proven to have key roles during mitotic processes in human embryonic stem cells (hESCs) (Ohgushi et al. 2017). We speculate that abnormal methylation of ABR may result in fetal growth retardation by influencing the mitotic processes of hESCs leading to RM.

Based on the results of our analysis of 1799 DMRs screened using the decidua samples from patients with RM and healthy pregnant women, $I S G 15$ was found to be the most relevant gene in RM. ISG15 is one of the several proteins induced by conceptus-derived type I or II interferons (IFNs) in the uterus and is implicated as an important factor in determining uterine receptivity to embryos in ruminants (Zhao et al. 2016). Further, ISG15 is involved in early bovine embryonic development and regulates IFNT expression in the blastocyst (Zhao et al. 2016). Thus, it is a candidate gene playing an important role during pregnancy through fetal growth.

To more broadly explore the potential function of the genes by the RM-related DMRs, we conducted a GO enrichment analysis. The GO enrichment analysis revealed that the DMRs were significantly enriched in response to system, multicellular organismal, and anatomical structure developments, the function of which is mostly performed by related genes, such as $H L A-G$ and $H L A-E$. HLA-G belongs to the non-classical HLA class I antigens and is a tolerogenic molecule that acts on the cells related to both innate and adaptive immunities (Verloes et al. 2017). Besides its immunosuppressive function in transplantation, $H L A-G$ expression is involved in implantation and protection of the semi-allogeneic fetus from the maternal immune system (Carosella et al. 2008; Hunt et al. 2005; Rebmann et al. 2007). Studies have proposed that $H L A-G$ plays an important role in early embryonic development (Yao et al. 2014). HLA-E products (class Ib human 
220

221

224

226

227

228

229

230

231

232

233

234

235

236

237

238

239

240

leukocyte antigens) act in the immunology of human reproduction as modulators of the maternal immune system during pregnancy (Gelmini et al. 2016). Recent studies have shown the functions of the HLA-E molecule and possible interactions with HLA-G. The relevance of $H L A-G$ and $H L A$ $E$ expression in the maternal-fetal interface seems to be regarding the inhibition of NK cellmediated lysis and possible influence on cytokine profiles (Gelmini et al. 2016). Pregnancy is a condition where women undergo major physiological and immunological alterations (Mishra et al. 2019), which are likely to be influenced or controlled by abnormal methylation levels of HLA-G and HLA-E.

A KEGG pathway enrichment analysis was employed to visualize the DMRs enriched for any pathways. CAMs, type I diabetes mellitus, and ECM-receptor interaction were found to be the frequently enriched pathways with several enriched genes, suggesting the potential roles of these genes in RM. For these pathways, $A L C A M, H L A-G$, and $H L A-E$ were the most related genes.

ALCAM is a member of the neuronal immunoglobulin-like domain superfamily of CAMs and promotes cell adhesion and signaling (Corbel et al. 1996; DeBernardo \& Chang 1996). It has been elucidated that $A L C A M$ is required for proper nephrogenesis and functions downstream of FZD3 during embryonic kidney development (Cizelsky \& Tata 2014). Thus, ALCAM is thought to play an important role in part of embryonic development and is also associated with fetal growth abnormalities.

These findings show that the abnormal methylation pattern of candidate genes may affect the stability of normal pregnancy and participate in the mechanisms that lead to RM. However, additional genetic and environmental factors might also play a role in the methylation patterns. 
241 We detected the mRNA expression levels of the candidate genes, ISG15, ABR, HLA-G, HLA-E, 242 and $A L C A M$. The association between the candidate genes and RM has been described earlier in this manuscript. The mRNA expression levels of the candidate genes were significantly higher in the RM group than in the control group. We speculate that the occurrence of RM might be related to the increase in mRNA expression levels of $I S G 15, A B R, H L A-G, H L A-E$, and $A L C A M$ in the decidua. In addition, the methylation level of $H L A-G$ and $H L A-E$ was lower in the RM group than in the control group. Hypermethylation inhibits gene expression. Therefore, we proposed that a decrease in the methylation level of HLA-G and HLA-E may increase mRNA expression, while an increase in the maternal $H L A-G$ and $H L A-E$ mRNA expression levels may affect fetal formation and development through an immune response and ultimately lead to RM.

251

\section{Conclusions}

In conclusion, this study first analyzed the DNA methylation status and mRNA expression levels of ISG15, ABR, HLA-G,HLA-E, and $A L C A M$ in decidua samples from patients with RM and healthy pregnant women. These five novel genes, all relevant to embryonic development, are likely to play a significant role in RM. Changes in the methylation and mRNA levels of these five genes may lead to the same abnormal embryonic gene expression, resulting in the blockage of embryonic or fetal formation and development and eventually leading to RM. Therefore, we hypothesized that $I S G 15, A B R, H L A-G, H L A-E$, and $A L C A M$ may be potential candidates for the progress and development of RM, which may also serve as new targets in the diagnosis of RM. While our results provide a direction for future research, limitations still exist in the present study. There is a possibility that this abnormal methylation is not the cause but a consequence of the defect that 
262 leads to RM. Further studies using large sample sizes are needed to validate the biological

263 functions and molecular mechanism of these genes. 


\section{Acknowledgments}

266 We thank all the members for their generous participation. 


\section{References}

Ashburner M, Ball CA, Blake JA, Botstein D, Butler H, Cherry JM, Davis AP, Dolinski K, Dwight SS, Eppig JT, Harris MA, Hill DP, Issel-Tarver L, Kasarskis A, Lewis S, Matese JC, Richardson JE, Ringwald M, Rubin GM, and Sherlock G. 2000. Gene ontology: tool for the unification of biology. The Gene Ontology Consortium. Nat Genet 25:25-29. 10.1038/75556

Banting GS, Barak O, Ames TM, Burnham AC, Kardel MD, Cooch NS, Davidson CE, Godbout R, McDermid HE, and Shiekhattar R. 2005. CECR2, a protein involved in neurulation, forms a novel chromatin remodeling complex with SNF2L. Hum Mol Genet 14:513-524. 10.1093/hmg/ddi048

Bestor TH. 2000. The DNA methyltransferases of mammals. Hum Mol Genet 9:2395-2402.

Bird AP, and Wolffe AP. 1999. Methylation-induced repression--belts, braces, and chromatin. Cell 99:451-454.

Brown LY, Bonney EA, Raj RS, Nielsen B, and Brown S. 2013. Generalized disturbance of DNA methylation in the uterine decidua in the CBA/J x DBA/2 mouse model of pregnancy failure. Biol Reprod 89:120. 10.1095/biolreprod.113.113142

Carosella ED, Favier B, Rouasfreiss N, Moreau P, and Lemaoult J. 2008. Beyond the increasing complexity of the immunomodulatory HLA-G molecule. Blood 111:4862.

Chen J, Morosan-Puopolo G, Dai F, Wang J, and Brand-Saberi B. 2010. Molecular cloning of chicken Cecr2 and its expression during chicken embryo development. Int J Dev Biol 54:925-929. 10.1387/ijdb.092933jc

Cizelsky W, and Tata A. 2014. The Wnt/JNK signaling target gene alcam is required for embryonic kidney development. Development 141:2064-2074.

Corbel C, Pourquie O, Cormier F, Vaigot P, and Le Douarin NM. 1996. BEN/SC1/DM-GRASP, a homophilic adhesion molecule, is required for in vitro myeloid colony formation by avian hemopoietic progenitors. Proc Natl Acad Sci U S A 93:2844-2849.

DeBernardo AP, and Chang S. 1996. Heterophilic interactions of DM-GRASP: GRASP-NgCAM interactions involved in neurite extension. J Cell Biol 133:657-666.

Draghici S, Khatri P, Tarca AL, Amin K, Done A, Voichita C, Georgescu C, and Romero R. 2007. A systems biology approach for pathway level analysis. Genome Res 17:1537-1545. 10.1101/gr.6202607

Esmailpour T, and Huang T. 2012. Defining new roles for TBX3 in human ESCs: TBX3 promotes human embryonic stem cell proliferation and neuroepithelial differentiation in a differentiation stage-dependent manner. Stem Cells 30:2152-2163.

Gelmini GF, Costa CH, Nardi FD, Wowk PF, Mattar SB, Schuffner A, Bicalho MD, and Roxo VM. 2016. Is HLA-E a possible genetic marker relevant for natural conception? American Journal of Reproductive Immunology 76:439-442.

Gene Ontology C. 2006. The Gene Ontology (GO) project in 2006. Nucleic Acids Res 34:D322-326. 10.1093/nar/gkj021

Griebel CP, Halvorsen J, Golemon TB, and Day AA. 2005. Management of spontaneous abortion. Am Fam Physician 72:1243-1250.

Hanna CW, McFadden DE, and Robinson WP. 2013. DNA methylation profiling of placental villi from karyotypically normal miscarriage and recurrent miscarriage. Am J Pathol 182:2276-2284. 10.1016/j.ajpath.2013.02.021

Horn KH, Warner DR, Pisano M, and Greene RM. 2011. PRDM16 expression in the developing mouse embryo. Acta Histochemica 113:150-155. 
Hou W, Li Z, Li Y, Fang L, Li J, Huang J, Li X, and You Z. 2016. Correlation between protein expression of FOXP3 and level of FOXP3 promoter methylation in recurrent spontaneous abortion. Journal of Obstetrics \& Gynaecology Research 42:1439-1444.

Hunt JS, Petroff MG, Mcintire RH, and Ober C. 2005. HLA-G and immune tolerance in pregnancy. Faseb Journal Official Publication of the Federation of American Societies for Experimental Biology 19:681.

Kanehisa M, and Goto S. 2000. KEGG: kyoto encyclopedia of genes and genomes. Nucleic Acids Res 28:27-30.

Kanehisa M, Goto S, Kawashima S, Okuno Y, and Hattori M. 2004. The KEGG resource for deciphering the genome. Nucleic Acids Res 32:D277-280. 10.1093/nar/gkh063

Kim JW, Park SY, Kim YM, Kim JM, Han JY, and Ryu HM. 2004. X-chromosome inactivation patterns in Korean women with idiopathic recurrent spontaneous abortion. $J$ Korean Med Sci 19:258-262. 10.3346/jkms.2004.19.2.258

Li E. 2002. Chromatin modification and epigenetic reprogramming in mammalian development. Nat Rev Genet 3:662673. $10.1038 / \mathrm{nrg} 887$

Li E, Bestor TH, and Jaenisch R. 1992. Targeted mutation of the DNA methyltransferase gene results in embryonic lethality. Cell 69:915-926.

Li TC, Makris M, Tomsu M, Tuckerman E, and Laird S. 2002. Recurrent miscarriage: aetiology, management and prognosis. Hum Reprod Update 8:463-481.

Mishra J, Talwar S, Kaur L, Chandiok K, Yadav S, Puri M, Sachdeva MP, Lakshmy R, and Saraswathy KN. 2019. Differential global and MTHFR gene specific methylation patterns in preeclampsia and recurrent miscarriages: A case-control study from North India. Gene 704:68-73. 10.1016/j.gene.2019.04.036

Ohgushi M, Minaguchi M, Eiraku M, and Sasai Y. 2017. A RHO Small GTPase Regulator ABR Secures Mitotic Fidelity in Human Embryonic Stem Cells. Stem Cell Reports 9:58.

Pildner vSS, and Ktm S. 2009. Recurrent Spontaneous Abortions- An Update on Diagnosis and Management. Journal Für Reproduktionsmedizin Und Endokrinologie 6:129-132.

Rai R, and Regan L. 2006. Recurrent miscarriage. Lancet 368:601-611. 10.1016/S0140-6736(06)69204-0

Rebmann V, Wagner S, and Grossewilde H. 2007. HLA-G expression in malignant melanoma. Seminars in Cancer Biology 17:422-429.

Reik W, and Walter J. 2001. Genomic imprinting: parental influence on the genome. Nat Rev Genet 2:21-32. $10.1038 / 35047554$

Smyth GK. 2005. limma: Linear Models for Microarray Data. Bioinformatics \& Computational Biology Solutions Using $R$ \& Bioconductor:397--420.

Takai D, and Jones PA. 2002. Comprehensive analysis of CpG islands in human chromosomes 21 and 22. Proc Natl Acad Sci U S A 99:3740-3745. 10.1073/pnas.052410099

Verloes A, Spits C, Vercammen M, Geens M, Lemaoult J, Sermon K, Coucke W, and Van dVH. 2017. The role of methylation, DNA polymorphisms and microRNAs on HLA-G expression in human embryonic stem cells. Stem Cell Research 19.

Yagi S, Hirabayashi K, Sato S, Li W, Takahashi Y, Hirakawa T, Wu G, Hattori N, Hattori N, Ohgane J, Tanaka S, Liu XS, and Shiota K. 2008. DNA methylation profile of tissue-dependent and differentially methylated regions (T-DMRs) in mouse promoter regions demonstrating tissue-specific gene expression. Genome Res 18:1969-1978. 10.1101/gr.074070.107

Yao GD, Shu YM, Shi SL, Peng ZF, Song WY, Jin HX, and Sun YP. 2014. Expression and Potential Roles of HLA- 
$349 \mathrm{G}$ in Human Spermatogenesis and Early Embryonic Development. PLoS One 9:e92889.

350

351

352

353

354

355

356

357

358

359
Yu M, Du G, Xu Q, Huang Z, Huang X, Qin Y, Han L, Fan Y, Zhang Y, Han X, Jiang Z, Xia Y, Wang X, and Lu C. 2018. Integrated analysis of DNA methylome and transcriptome identified CREB5 as a novel risk gene contributing to recurrent pregnancy loss. EBioMedicine 35:334-344. 10.1016/j.ebiom.2018.07.042

Zhao S, Wu Y, Gao H, Evans A, and Zeng SM. 2016. Roles of interferon-stimulated gene 15 protein in bovine embryo development. Reprod Fertil Dev. 10.1071/RD15209

Ziller MJ, Gu H, Muller F, Donaghey J, Tsai LT, Kohlbacher O, De Jager PL, Rosen ED, Bennett DA, Bernstein BE, Gnirke A, and Meissner A. 2013. Charting a dynamic DNA methylation landscape of the human genome. Nature 500:477-481. 10.1038/nature12433 


\section{Figure 1}

Viewsof methylation variable positions (MVPs) result.

(a) Volcano plot of MVPs between control and RM. Each dot represents an individual MVPs. Dots that showed $\mathrm{P}$ values $<=0.01$ after Benjamini \& Hochberg correction are colored red while $P$ values $>=0.01$ after Benjamini $\&$ Hochberg correction are colored black. The $x$-axis represents $\log 2$ (Fold Change). The y-axis shows the - $\log 10$ ( $p$ value).(b) Heat map of MVPs between control and RM. Each row represents a locus and each column represents a sample. Red indicates that the methylation level is up-regulated and blue indicates down-regulated methylation level. 

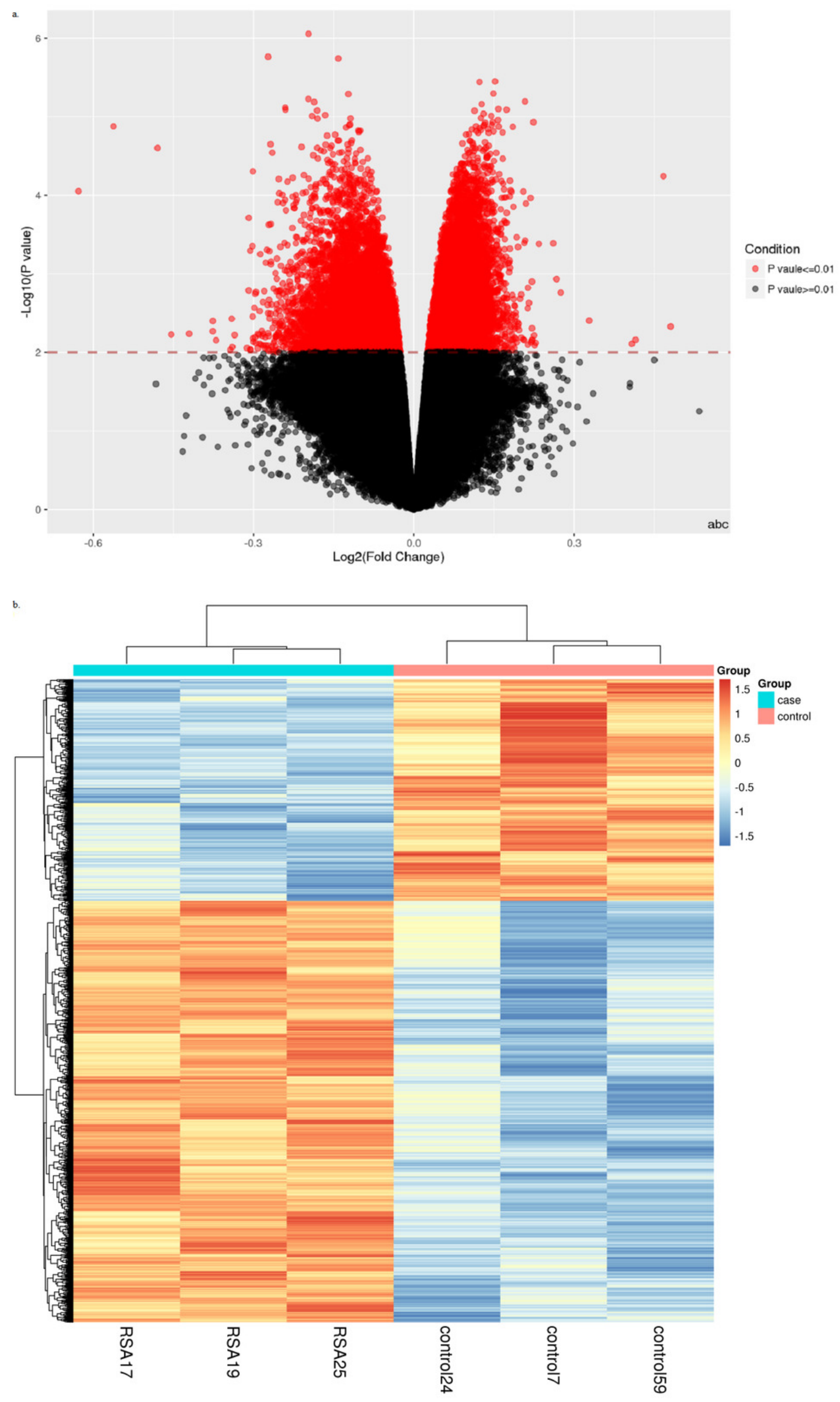


\section{Figure 2}

GO enrichment analysis and GO-Tree of differentially methylated regions (DMRs).

(a) GO enrichment analysis. The horizontal axis was the value of -log(P-value). The vertical axis was GO terms colored in three levels. The most significantly enriched terms for each level are shown. Red represented biological process (BP), green represented cellular component (CC), and blue represented molecular function (MF).(b-d) GO-tree. Hierarchical trees of three categories represented biological process (BP), cellular component (CC) and molecular function (MF) respectively. Colored squares represented the most enriched GO terms. 

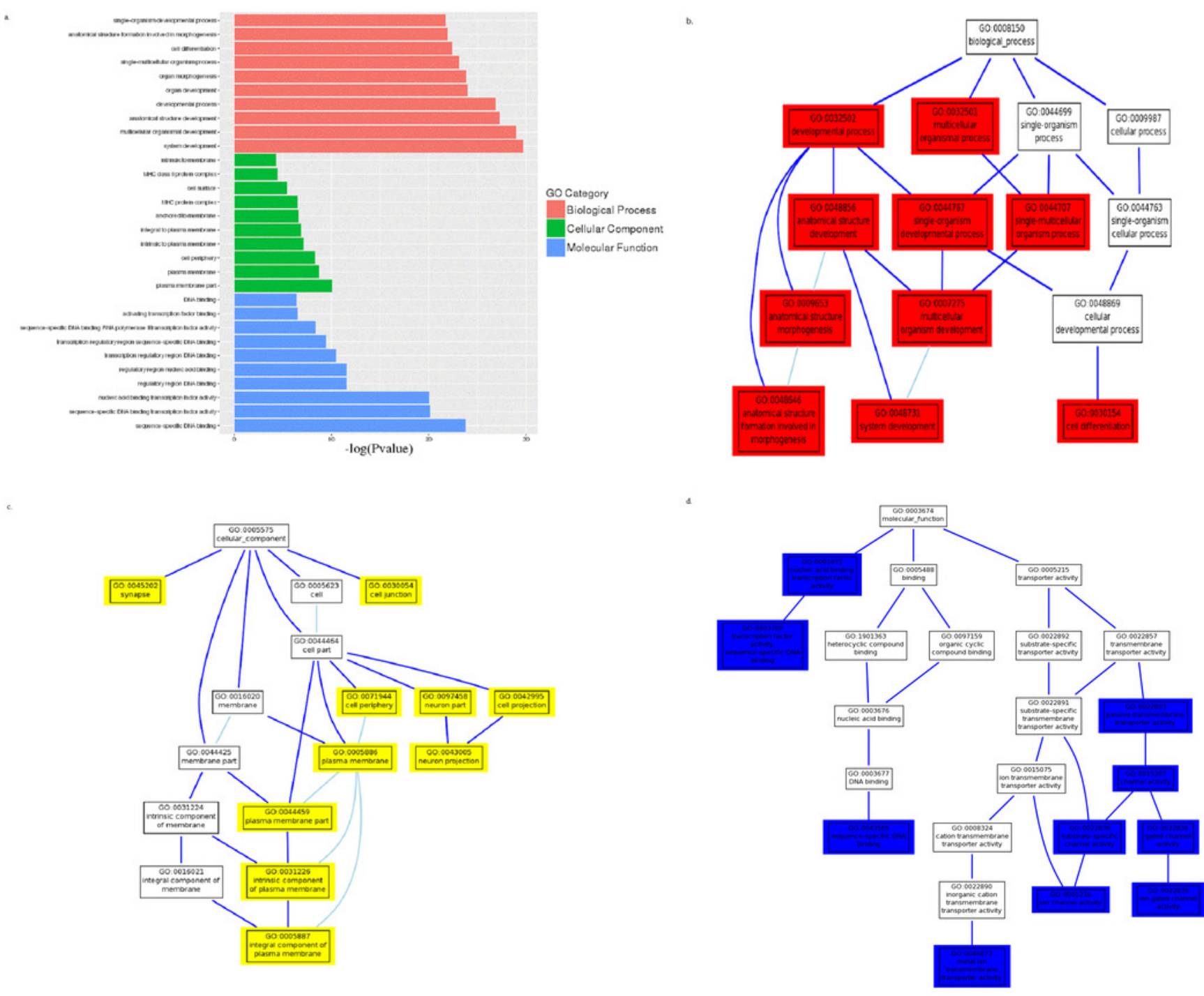


\section{Figure 3}

Pathway Enrichment Analysis of differentially methylated regions (DMRs).

KEGG pathway enrichment analysis. The horizontal axis was the top 20 most significantly enriched KEGG pathway. The vertical axis was the value of - $\log (\mathrm{P}$-value).

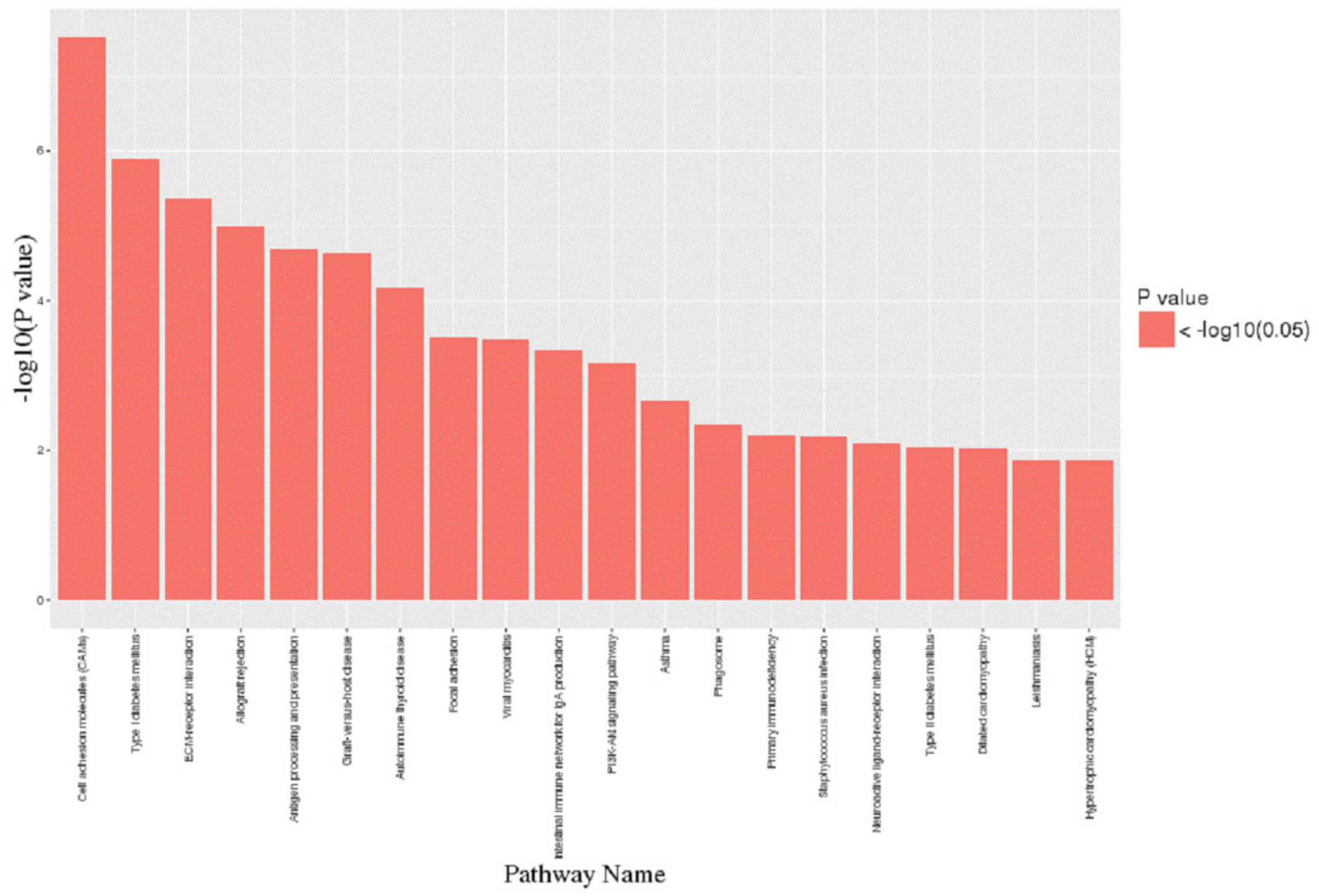


Figure 4

Comparisonof mRNA expression levels of candidate genes in decidua tissues.

$\mathrm{n}=3$, mean $\pm S D$, and independent sample T-test was used for comparison among groups.

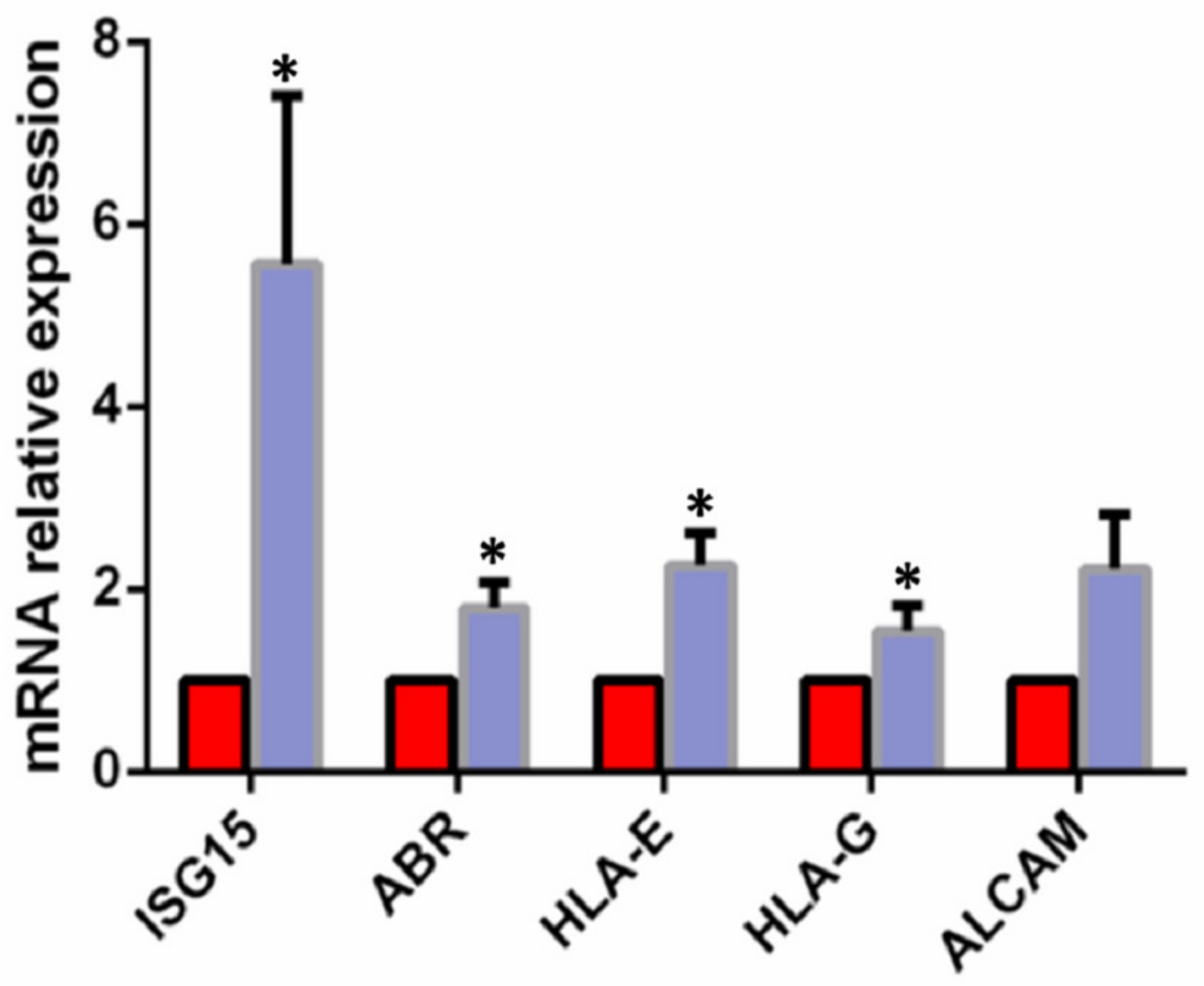

$\square$ control case 


\section{Table $\mathbf{1}$ (on next page)}

Clinical characteristics of the recruited recurrent miscarriage (RM) patients $(n=15)$ and normal pregnant (NP) women $(n=15)$ 
1 Table 1. Clinical characteristics of the recruited recurrent miscarriage $(\mathrm{RM})$ patients $(\mathrm{n}=15)$ and

2 normal pregnant (NP) women $(\mathrm{n}=15)$.

3

\begin{tabular}{ccc}
\hline & RM (mean \pm SD) & NP (mean \pm SD) \\
\hline Age(years) & $30.13 \pm 4.42$ & $29.53 \pm 6.65$ \\
Gestational weeks & $8.27 \pm 1.87$ & $7.33 \pm 0.82$ \\
Childbearing history & $0.27 \pm 0.46$ & $0.53 \pm 0.52$ \\
Spontaneous abortion history & $2.53 \pm 0.64$ & - \\
\hline
\end{tabular}

4

5 
Table 2 (on next page)

The methylation variable positions (MVPs) 
1 Table 2. The methylation variable positions (MVPs)

\begin{tabular}{lllll}
\hline gene & probeID & $\operatorname{logFC}$ & $\mathrm{t}$ & P.Value \\
\hline C2orf54 & $\operatorname{cg} 06546066$ & 0.209665031 & -23.50980689 & $8.83141 \mathrm{E}-07$ \\
CECR2 & $\operatorname{cg} 27415324$ & -0.260083489 & -20.80755786 & $1.7299 \mathrm{E}-06$ \\
TMEM161B & $\operatorname{cg} 18295770$ & 0.101275636 & -20.62487447 & $1.81586 \mathrm{E}-06$ \\
ABR & $\operatorname{cg} 09639964$ & -0.590951212 & 18.25084313 & $3.55356 \mathrm{E}-06$ \\
EMCN & $\operatorname{cg} 13149566$ & 0.419028173 & 18.19585048 & $3.61283 \mathrm{E}-06$ \\
TBCD & $\operatorname{cg} 06218079$ & -0.051208796 & 17.10132934 & $5.07438 \mathrm{E}-06$ \\
GP5 & $\operatorname{cg} 10658438$ & 0.216737391 & -17.05295333 & $5.15363 \mathrm{E}-06$ \\
PRICKLE2-AS3 & $\operatorname{cg} 05063999$ & -0.013472989 & -16.61485758 & $5.94177 \mathrm{E}-06$ \\
NBPF22P & $\operatorname{cg} 12475092$ & 0.007591767 & 16.38637216 & $6.40879 \mathrm{E}-06$ \\
TBX3 & $\operatorname{cg} 17070988$ & 0.562046907 & -16.33297277 & $6.52408 \mathrm{E}-06$ \\
\hline
\end{tabular}

2

3

4

5

6

7

8

9

10

11 


\section{Table 3 (on next page)}

Gene ontology (GO) Enrichment analysis (top 3 significantly enriched biology terms) 
1 Table 3. Gene ontology (GO) Enrichment analysis (top 3 significantly enriched biology terms) 2

\begin{tabular}{llll}
\hline ID & Category & Term & p-value \\
\hline biological process (BP) & GO:0048731 & system development & $1.86 \mathrm{E}-30$ \\
& GO:0007275 & multicellular organismal development & $1.05 \mathrm{E}-29$ \\
& GO:0048856 & anatomical structure development & $4.62 \mathrm{E}-28$ \\
\hline cellular component (CC) & GO:0044459 & plasma membrane part & $9.42 \mathrm{E}-11$ \\
& GO:0005886 & plasma membrane & $1.86 \mathrm{E}-09$ \\
& GO:0071944 & cell periphery & $4.57 \mathrm{E}-09$ \\
\hline molecular function (MF) & GO:0043565 & sequence-specific DNA binding & $1.61 \mathrm{E}-24$ \\
& GO:0003700 & sequence-specific DNA binding transcription factor activity & $7.89 \mathrm{E}-21$ \\
& GO:0001071 & nucleic acid binding transcription factor activity & $9.29 \mathrm{E}-21$ \\
\hline
\end{tabular}


Table 4 (on next page)

GO-related genes 


\begin{tabular}{lll}
\hline GO_term & GO_category & symbols_in_list \\
& & \\
system development & HES4,PRKCZ,SKI,PRDM16,ALPL,RUNX3,TAL1,TTLL7, \\
& biological_process & BARHL2,OLFM3,COL11A1,TBX15,WARS2,HLA-G \\
plasma membrane part & & TNFRSF4,PRKCZ,AJAP1,SYTL1,OPRD1,PTPRU,1L12RB2, \\
& cellular_component & GJA5,GNG4,GPR137B,SNTG2,TPO,HLA-G,HLA-E \\
sequence-specific DNA binding & & \\
\hline
\end{tabular}

3 


\section{Table 5 (on next page)}

KEGG enrichment analysis (top 10 significantly enriched pathway terms) 
1 Table 5. KEGG enrichment analysis (top 10 significantly enriched pathway terms)

\begin{tabular}{llll}
\hline ID & Category & Term & p-value \\
\hline KEGG PATHWAY & PATHWAY:hsa04514 & Cell adhesion molecules (CAMs) & $3.00 \mathrm{E}-08$ \\
& PATHWAY:hsa04940 & Type I diabetes mellitus & $1.27 \mathrm{E}-06$ \\
& PATHWAY:hsa04512 & ECM-receptor interaction & $4.24 \mathrm{E}-06$ \\
& PATHWAY:hsa05330 & Allograft rejection & $1.02 \mathrm{E}-05$ \\
& PATHWAY:hsa04612 & Antigen processing and presentation & $2.05 \mathrm{E}-05$ \\
& PATHWAY:hsa05332 & Graft-versus-host disease & $2.32 \mathrm{E}-05$ \\
& PATHWAY:hsa05320 & Autoimmune thyroid disease & $6.56 \mathrm{E}-05$ \\
PATHWAY:hsa04510 & Focal adhesion & $3.02 \mathrm{E}-04$ \\
PATHWAY:hsa05416 & Viral myocarditis & $3.34 \mathrm{E}-04$ \\
& PATHWAY:hsa04672 & Intestinal immune network for IgA production & $4.50 \mathrm{E}-04$ \\
\hline
\end{tabular}


Table 6(on next page)

KEGG-related genes 
Table 6. KEGG-related genes

\begin{tabular}{lll}
\hline Pathway_name & Pathway_class & Symbols_in_list \\
\hline $\begin{array}{lll}\text { Cell adhesion molecules } \\
\text { (CAMs) }\end{array}$ & Signaling molecules and interaction & CADM3,F11R,NRXN1,ITGA4,PDCD1,ALCAM,NLGN1,CLDN16, \\
Type I diabetes mellitus & Endocrine and metabolic diseases & HLA-G,HLA-E,HLA-B,HLA-DQB1,HLA-DOB,HLA-DMB \\
& & DMB,HLA-DMA,HLA-DOA,HLA-DPB1,PTPRN2,PRF1,INS \\
Allograft rejection & Immune diseases & HLA-G,HLA-E,HLA-B,TNF,HLA-DQB1,HLA-DOB, \\
& & HLA-DMB,HLA-DMA,HLA-DOA,HLA-DPB1,PRF1 \\
Antigen processing & Immune system & NFYC,HLA-G,HLA-E,HLA-B,TNF,HLA-DQB1, \\
and presentation & & HLA-DOB,TAP2,TAP1,HLA-DMB,HLA-DMA,HLA-DOA \\
Focal adhesion & COL11A1,LAMB3,ITGA4,COL6A3,ITGA2,PDGFRB,FLT4,MYLK4 3.02E-04 \\
& & TNXB,COL11A2,THBS2,EGFR,ITGA8,DOCK1,CCND1,CCND2 \\
\hline
\end{tabular}

